\title{
High resolution crystal structure of the FAK FERM domain reveals new insights on the Druggability of tyrosine 397 and the Src $\mathrm{SH} 3$ binding site
}

Timothy Marlowe $e^{1,2,6^{*}}$ D, Alexey Dementiev ${ }^{3,4}$, Sheila Fige ${ }^{5}$, Andrew Rivera ${ }^{1,6}$, Michael Flavin ${ }^{3}$ and William Cance ${ }^{1,6^{*}}$

\begin{abstract}
Background: Focal Adhesion Kinase (FAK) is a major cancer drug target that is involved in numerous aspects of tumor progression and survival. While multiple research groups have developed ATP-competitive small molecule inhibitors that target the kinase enzyme, recent attention has been focused on the FAK FERM (Band 4.1, Ezrin, Radixin, Moesin) domain that contains key residue Y397 and contributes to many protein-protein interactions. Previous $x$-ray crystal structures of the FAK FERM domain gave conflicting results on the structure of the Y397 region and therefore the overall druggability.
\end{abstract}

Results: Here, we report the identification of a higher resolution crystal structure of the avian FAK FERM domain that shows conformational differences in Y397 and surrounding residues in the F1 lobe. In addition, we resolve the residues of the Src SH3 binding site, an area of the FERM domain that has previously shown limited electron density.

Conclusions: These crystallographic data suggest that the Y397 region is highly dynamic and question the druggability of a putative pocket on the F1 lobe. In addition, new electron density data around the Src SH3 binding site provide structural insight on the FAK-Src activation cascade through a putative auto-inhibitory conformation.

Keywords: Focal adhesion kinase, FERM domain, Drug discovery

\section{Background}

Focal Adhesion Kinase (FAK) is a $125 \mathrm{kDa}$ non-receptor tyrosine kinase that has multiple implications in cancer cell signaling, invasion, proliferation, and metastasis $[1,2]$. In addition, FAK is overexpressed in many invasive solid tumors while minimally expressed in normal tissue, making it an attractive therapeutic target for cancer treatment [3-5]. Activation of FAK occurs not only through autophosphorylation of key residue tyrosine 397 (Y397) by FAK itself via the kinase enzyme [6], but also through transphosphorylation by receptor tyrosine kinases which can bind to the FAK N-terminal FERM domain directly

\footnotetext{
* Correspondence: tmarlowe@email.arizona.edu; wcance@email.arizona.edu Synopsis A new x-ray crystal structure of the FAK FERM domain identifies differences in both the Y397 region and the Src SH3 binding site.

${ }^{1}$ Interdisciplinary Oncology, University of Arizona College of Medicine -

Phoenix, 475 N 5th Street, Phoenix, AZ 85004, USA

Full list of author information is available at the end of the article
}

$[7,8]$. The proto-oncogene Src directly binds to FAK through its $\mathrm{SH} 2$ and $\mathrm{SH} 3$ domains at phosphorylated FAK Y397 and a proline-rich region in the FERM-Kinase linker, respectively $[9,10]$. Src-FAK binding promotes FAK phosphorylation at Y576, Y577, Y861, Y925, and thus the full activation of FAK oncogenic signaling [11, 12]. FAK also functions as an intracellular scaffolding protein through its FERM domain and C-terminal FAT domain regions where FAK forms multiple protein-protein interactions to connect oncogenic signaling pathways [13, 14]. Many efforts have been made to therapeutically target FAK at the catalytic ATP-binding region of the protein (kinase enzyme) and several candidates have entered clinical trials for the treatment of various solid tumors [15-18]. Despite the high-potency of these kinase inhibitors in vitro, results from clinical trials have been disappointing due to multiple factors such as lack of a predictive biomarker,

(C) The Author(s). 2019 Open Access This article is distributed under the terms of the Creative Commons Attribution 4.0 International License (http://creativecommons.org/licenses/by/4.0/), which permits unrestricted use, distribution, and 
compensatory resistance mechanisms to kinase inhibitors, and lack of targeting key FAK scaffolding regions [19, 20].

As an alternative approach to target FAK, several groups have attempted targeting the FAK FERM domain at a binding site proximal to key residue Y397 as a means to target the FAK activation site directly and inhibit compensatory FAK phosphorylation via transphosphorylation [21-24]. However, significant challenges remain due to the low druggability of the FAK FERM domain and the dynamic nature of the Y397 FERM-Kinase linker region. Previous x-ray crystal structures of the avian FERM domain (2AL6, $2.35 \AA$ ) [25] and the human FERM domain (4NY0, $2.8 \AA$ ) [26] yielded conflicting results regarding the electron density of residues surrounding Y397. In structure 2AL6, the segment from residue 394-403 can be detected bound to the F1 lobe in a proposed "autoinhibited" state, however in structure 4NY0 there is no clear density of segment 394-403. Structure 2AL6 contains adequate electron density to position 394-403 proximal to the F1 lobe. In addition, structure 2AL6 shows the appearance of a putative druggable pocket proximal to Y397 that has been exploited in multiple structure-based virtual screening campaigns, however this pocket is not visible in structure 4NY0. Nonetheless, it is still unclear whether the region surrounding FAK Y397 can be targeted with small molecules and whether FAK FERM x-ray crystal information can be reliably utilized to guide computer-aided drug design efforts.

Here, we report an x-ray crystal structure of the avian FAK FERM domain at a higher resolution $(1.97 \AA)$ than previously reported [25], elucidating new structural features of the FERM-Kinase linker segment that impact the druggability of the Y397 region. In addition, we identify a novel conformation of the Src SH3 binding site $\left({ }^{363} \mathrm{QKE}-\right.$ GERALPSIPK $^{375}$ ) that may have implications in FAK activation via Src. Finally, our crystal structure confirms a previous report identifying the site of dimerization (W266) between two FERM monomers [26].

\section{Results}

\section{Overall structure}

The crystal structure of the $\mathrm{N}$-terminal domain (residues 31-405, FERM domain) of the avian FAK was solved to a resolution of $1.97 \AA$ by molecular replacement method, using the structure of the FAK FERM (PDB code 2AL6) as search model (Table 1). The protein crystallized in the orthorhombic space group P 212121 with two molecules per asymmetric unit (Fig. 1). The final structure, refined with $R_{\text {work }}$ of 17.3 and $R_{\text {free }}$ of 21.2 , is more ordered than that of the search model [27]. Almost all residues are ordered in the electron density map except residues 376390 and 403-405 in molecule A and residues 31-33, 182$187,311-313,377-393$, and 403-405 in molecule B. The
Table 1 Data collection and refinement statistics for FERM structure: PDB code 6CB0

\begin{tabular}{|c|c|}
\hline \multicolumn{2}{|l|}{ Data collection } \\
\hline Wavelength $(\AA)$ & 0.9787 \\
\hline Resolution range $(\AA)$ & $123.90-1.97(2.08-1.97)$ \\
\hline Space group & P 212121 \\
\hline Unit cell & 50.15123.90135.16909090 \\
\hline Total reflections & 400,847 \\
\hline Unique reflections & $55,766(6816)$ \\
\hline Multiplicity & $7.2(7.2)$ \\
\hline Completeness (\%) & $92.43(78.50)$ \\
\hline Mean I/sigma (I) & $21.5(1.99)$ \\
\hline Wilson B-factor & 35.34 \\
\hline R-merge (\%) & $5.2(82.3)$ \\
\hline \multicolumn{2}{|l|}{ Refinement } \\
\hline Resolution range $(\AA)$ & 50.01-1.97 (2.02-1.97) \\
\hline Number Reflections/unique & 55,679 / 52,861 (4186) \\
\hline Completeness (\%) & $92.43(99.98)$ \\
\hline Mean I/sigma(I) & $19.8(2.0)$ \\
\hline Reflection used for Rfree (\%) & 5.1 \\
\hline R-work (\%) & $17.3(24.7)$ \\
\hline R-free (\%) & $21.2(29.0)$ \\
\hline Number of non-hydrogen atoms & 5890 \\
\hline Macromolecules & 5491 \\
\hline Water & 399 \\
\hline Protein residues & 700 \\
\hline RMS (bonds) & 0.019 \\
\hline RMS (angles) & 1.79 \\
\hline Ramachandran favored (\%) & 97.4 \\
\hline Ramachandran allowed (\%) & 2.5 \\
\hline Ramachandran outliers (\%) & 0.1 \\
\hline Clashscore & 2.59 \\
\hline Average B-factor & 45.35 \\
\hline Macromolecules & 45.06 \\
\hline Solvent & 49.30 \\
\hline
\end{tabular}

Statistics for the highest-resolution shell are shown in parentheses

previous crystal structure of the FAK FERM domain (4NY0) determined at $2.8 \AA$ resolution did not have adequate electron density for the critical Src SH3 binding motif (residues 363-375) to be visible. In this work, we solved the FERM structure at a much higher $1.97 \AA$ resolution and we were able to rebuild the segment (363-375) in this area including the RALPSIP motif. The average B factor values for $\mathrm{C} \alpha$ atoms of Lys375 and Val391 located at the $\mathrm{N}$ - and C-termini of this unconnected part are 62 $\AA^{2}$ and $77 \AA^{2}$, respectively. These values are relatively close to an average $B$ factor value for all protein atoms of the model is ( $45 \AA^{2}$ - Table 1), which indicates correct 


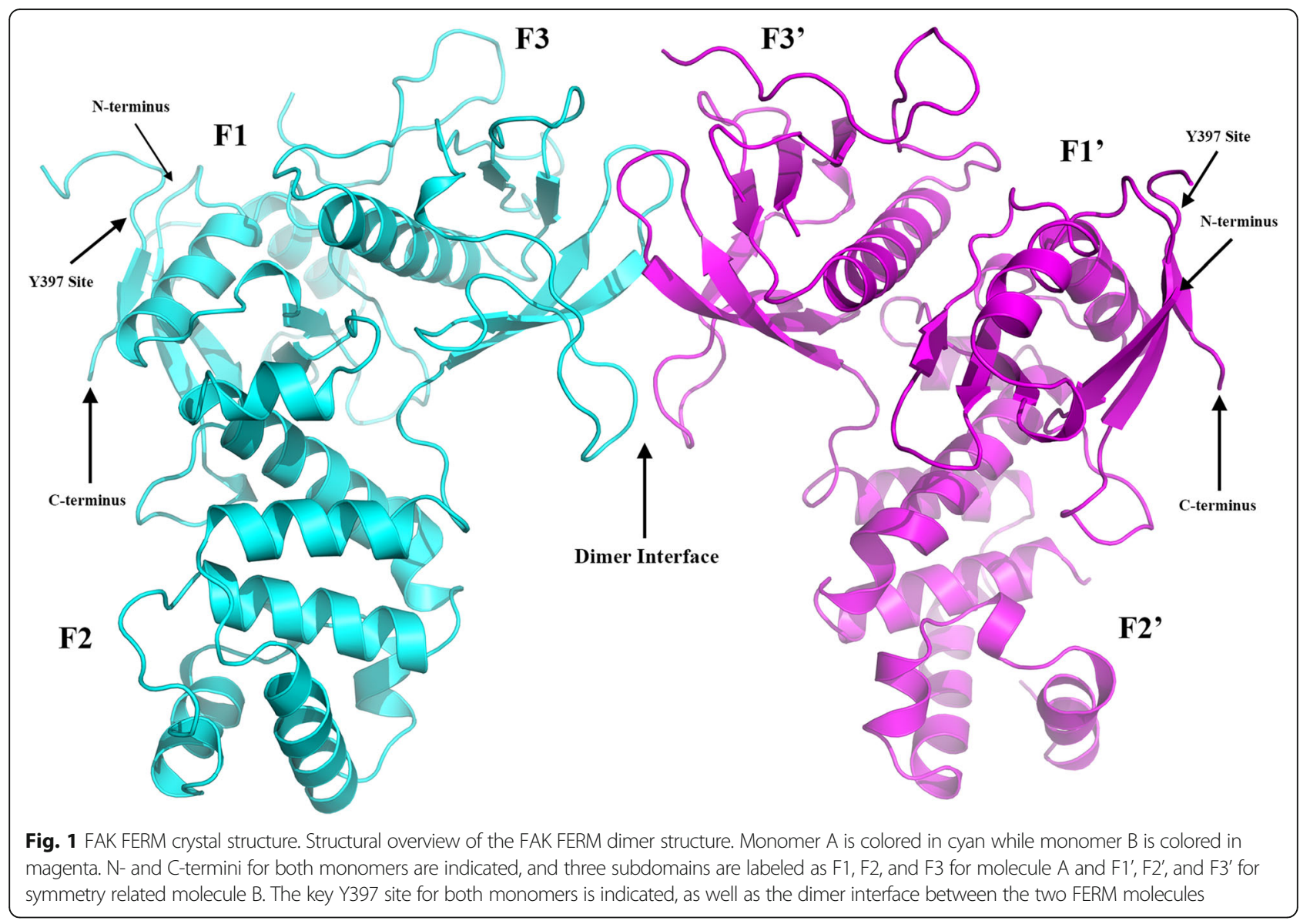

model rebuilding for the two segments flanking this unconnected part (residues 376-390). The $\mathrm{N}$-terminal 3 residues of the expression tag remnant for both FERM molecules could not be defined.

The overall conformation of the FERM molecule displays strong structural similarity with other FERM domains of ERM family members ezrin, radixin, and moesin, consisting of three subdomains (labeled F1, F2, and F3, Fig. 1). Optimal superposition of two FERM molecules in the asymmetric unit results in 2149 equivalent atoms with the RMSD values of $0.438 \AA$ (calculated in PyMOL). The major differences between the two molecules are observed in two loops in subdomains F2 and F3, which is very similar to the crystal structure of the search model PDB 2AL6. Also, similar to the search model, there is non-crystallographic symmetry for the FERM dimer in the crystal packing.

\section{Comparison between our structure at $1.97 \AA$ and previously published structures}

Next, we compared our FAK FERM crystal structure (PDB 6CB0) to previously published crystal structures at $2.35 \AA$ (PDB 2AL6) and $2.8 \AA$ (PDB 4NY0). We first started with sequence alignment, structural superposition, and RMSD calculation using PyMOL software (Fig. 2a). Structure 2AL6 aligned with 6 CB0 at a RMSD of $0.27 \AA$ and 2050 equivalent atoms. Structure 4 NY0 aligned with 6 CB0 at a RMSD of $0.709 \AA$ and 2143 equivalent atoms. Overall, all three FERM structures are quite similar other than key differences in the Y397 FERM-Kinase linker region (residues 391-402) and the Src SH3 binding site (residues 363-375). Our structure (6CB0) has unique density from residues 363-375 not present in other structures and shows a different conformation at residues 391-402. The average $\mathrm{B}$ factor for all atoms within region 363-375 and $391-402$ is $54.6 \AA^{2}$ and $59.0 \AA^{2}$, respectively. Also, there are minor differences in loop regions within the F3 lobe of all three FERM domains. Multiple sequence alignment of the three FERM domain proteins showed a high degree of sequence identity (96\%) and homology (99\%) between human and avian sequences (Fig. 2b). Intriguingly, regions 391-402 and 363-375 with major differences in electron density share $100 \%$ sequence identity.

Differences in region surrounding FAK Y397 and F1 lobe Upon further examination in PyMOL we noticed a significant difference in electron density of key activation residue Y397 and surrounding residues in the FERM-kinase 


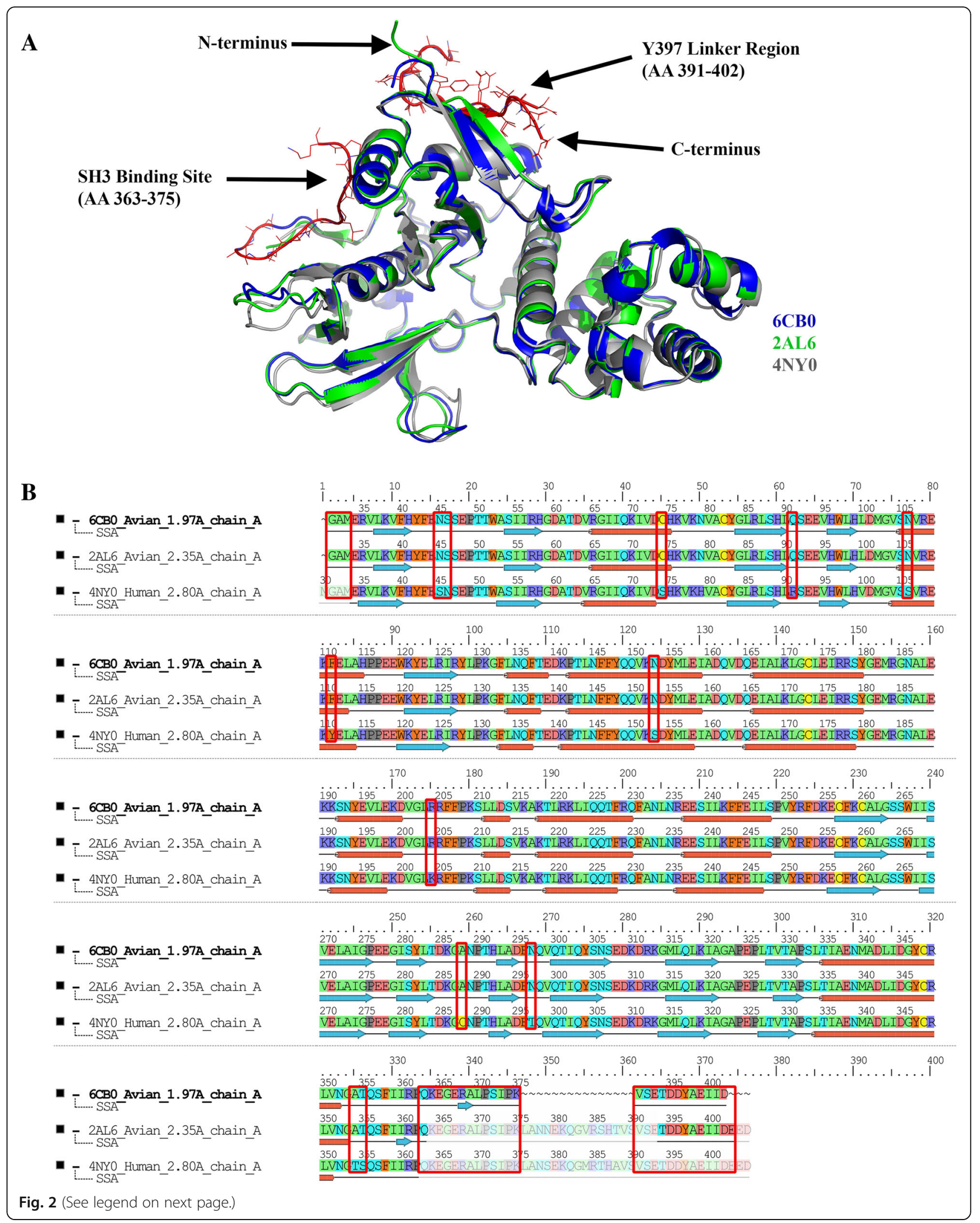


(See figure on previous page.)

Fig. 2 Alignment of our FAK FERM structure with previously published avian and human structures. a Structural alignment of our high resolution FERM structure (6CBO - blue) with published avian (2AL6 - green) and human (4NYO - grey) FERM structures. Alignment was performed in PyMOL software. Note, key regions in our structure that show unique differences in electron density are highlighted in red. b Multiple sequence alignment of FAK FERM structures 6CBO, 2AL6, and 2NY0 performed with the Schrodinger multiple sequence viewer tool. Residue coloring is based on side chain chemistry. Key regions of extra electron density and non-conserved residues between human and avian sequences are boxed in red

linker as well as residues in the F1 lobe proximal to Y397. In our structure, Y397 is rotated inwards toward the protein and occupies a pocket formed by R35, R57, and H58, however in PDB 2AL6, residue Y397 is pointed outward towards the solvent (Fig. 3a). Additionally, there are alternative conformations of residues G31, A32, M33, E34, R35, R57, and E109 which cause the disappearance of a pocket formed by these residues in PDB 2AL6 that has been previously exploited by computational drug discovery efforts (Fig. 3b). Furthermore, our structure shows unique differences in this area not previously observed in both chains of PDB 2AL6.

To calculate the potential druggability differences between our structure and PDB 2AL6, we subsequently utilized the Schrodinger SiteMap tool, which uses a grid-based searching algorithm around the solvent-exposed surface of the protein to predict enclosed regions that favor small molecule binding. SiteMap analysis of the Y397 region in PDB 2AL6 revealed a defined pocket with a SiteScore of 0.90 (a SiteScore $>0.8$ indicates a druggable site) whereas similar analysis of our $1.97 \AA$ structure resulted in the inability to predict any druggable site near Y397 (Fig. 3c and d). These results indicate a significant difference in computationally-predicted druggability of the Y397 region between the two FAK FERM structures.

\section{Novel conformation of the Src SH3 binding motif}

The previous crystal structure of the FAK FERM domain (4NY0) did not have adequate electron density for the critical Src SH3 binding motif (residues 363-375) to be visible. In our structure we have better resolution of this region $\left({ }^{363}\right.$ QKEGERALPSIPK $\left.{ }^{375}\right)$ and therefore have found a previously unidentified conformation of this area. As shown in Fig. 4, residues L370 and P371 of the SH3 binding motif from FERM molecule A pack into a pocket formed by residues I359, Y304, E338, M102, and H99 in between the F1 and F3 lobes of the protein. Residues 372-375 of the motif bind along the side of the F1 lobe however visibility of residues 376-390 cannot be determined. FERM molecule B has a different conformation more similar to previously published FERM structure (4NY0). Altogether, these data suggest an intramolecular interaction between the Src SH3 binding site and the FERM domain which may regulate the activity of FAK and downstream signaling.

\section{Discussion}

Several reports have been published focusing on the discovery of FAK inhibitors that directly target the FERM domain proximal to key residue Y397 as opposed to the ATP-binding pocket of the kinase domain [21-24]. These studies have all utilized computer-aided drug discovery methodology based on the previously published crystal structure of the avian FAK FERM domain (PDB 2AL6) and a putative pocket on the FERM F1 lobe nearby Y397. Here, we report a higher resolution x-ray crystal structure of the FAK FERM domain and reveal novel structural information of the protein that has implications on the druggability of FAK FERM nearby Y397. We identified an alternative conformation of Y397 and the residues encompassing a nearby pocket on the F1 lobe in our structure compared to the previously published structure (PDB 2AL6). Computational prediction of this pocket in our structure suggested that the Y397 region has poor druggable characteristics, contrasting previous reports [21-24]. We speculate that the Y397 region, which is part of the FERM-kinase linker and proximal to the F1 lobe, is highly dynamic in vivo and may represent a combination of conformations observed in our structure as well as previous structures. Furthermore, it is uncertain whether the Y397 region identified here and in other structures represents the biologically relevant conformation or a result of crystal packing. Further structural confirmation of the Y397 region by solution-based studies such as NMR is necessary to fully understand the structure and function of this critical region.

It is important to note that although previous FERM Y397 drug discovery efforts have identified candidate molecules with interesting cell-based data [21-24], there are no inhibitors to date with valid structural evidence of binding specificity (i.e. $x$-ray co-crystal structures, NMR HSQC chemical shift perturbation studies, etc.). In these studies, micromolar concentrations of compound were required for cellular effects on viability/apoptosis and on-target FAK inhibition was only measured by western blotting and in vitro kinase assays. Only one study [24] performed a biophysical assay (Bio-Layer Interferometry), using high concentrations of compound $(500 \mathrm{uM})$ and no report of binding affinity $\left(\mathrm{K}_{\mathrm{D}}\right)$. An explanation for the disconnect between biological activity and structural validation of FAK FERM inhibitors could be that FAK FERM 


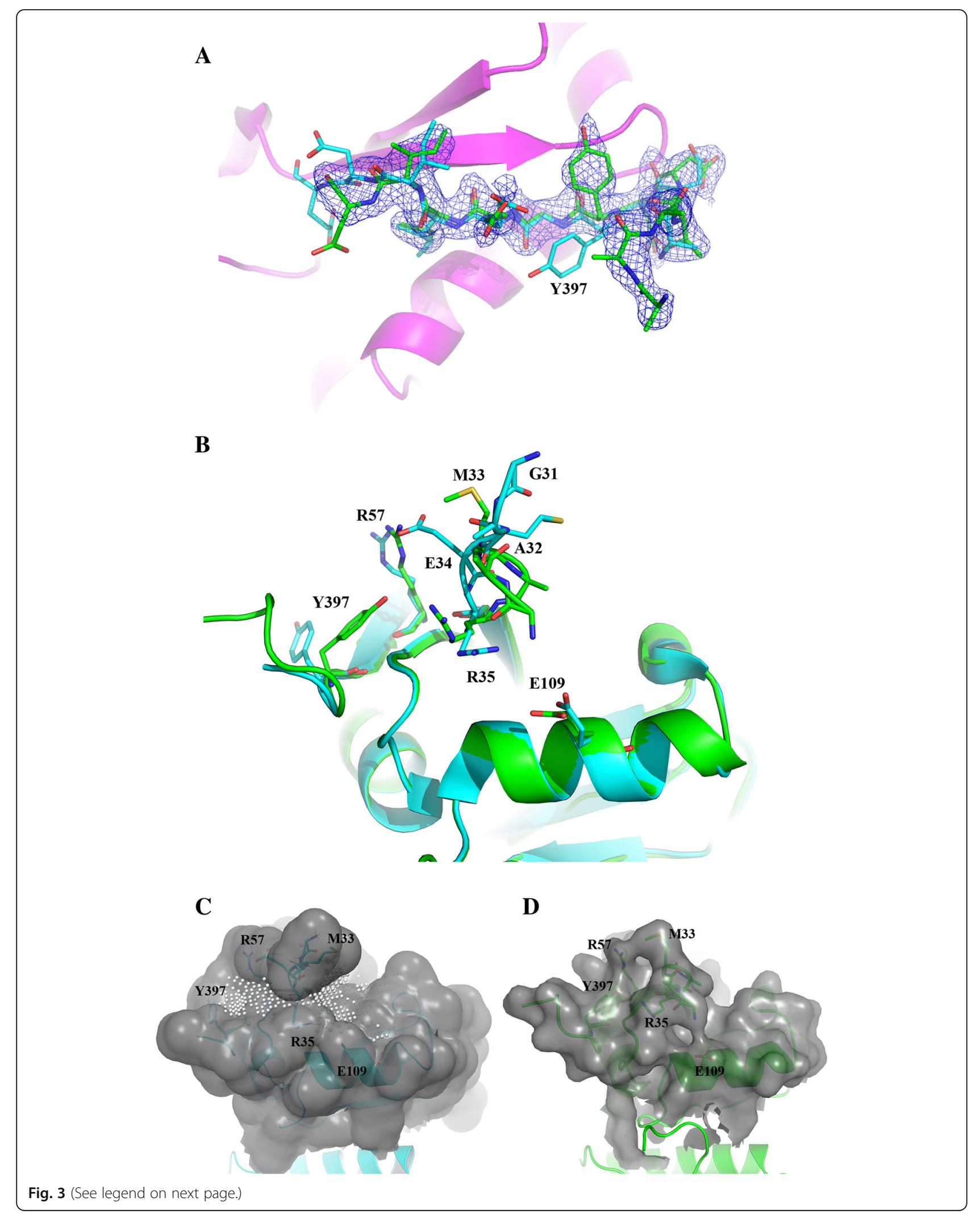


(See figure on previous page.)

Fig. 3 Difference in Y397 region between our structure and previously published FERM structure. a Comparison of Y397 within FERM-kinase linker region between our structure (green) and PDB 2AL6 (cyan). 2Fo-Fc electron density map contoured at $1.0 \sigma$ is shown in mesh. Note, common ribbon structure between both proteins is shown in magenta. $\mathbf{b}$ Comparison of FERM F1 lobe residues nearby Y397 in our structure (green) and PDB 2 AL6 (cyan). c Depiction of druggable pocket nearby Y397 in PDB 2AL6. Note, SiteScore = 0.9. d Depiction of region nearby Y397 in our FERM structure. Note, there is an absence of a discernible pocket and SiteMap did not identify this region as druggable

x-ray crystallography studies do not correctly sample the pharmacologically-active conformation required for small molecule binding. In addition, putative FAK FERM inhibitors may be indirectly acting on the FAK pathway through direct binding of an upstream molecule such as a transmembrane receptor. As such, our data bring concerns to the druggability of the Y397 region and suggest that structural confirmation of binding and alternative drug discovery/structural biology strategies (i.e. fragment screening, NMR, covalent tethering) rather than a sole computational approach based on $\mathrm{x}$-ray should be employed to find small molecule inhibitors of the Y397 region.

Another important finding of our FAK FERM structure was the visibility of the $\mathrm{Src} \mathrm{SH} 3$ binding site $\left({ }^{363}\right.$ QKEGERALPSIPK $\left.{ }^{375}\right)$, which was not found in previous FAK FERM structures $[25,26]$. In our structure, the Src SH3 binding site makes intramolecular contacts with a pocket in between the FERM F1 and F3 lobes. This finding may represent a novel allosteric form of regulation of FAK activation through modulation of the Src-FAK interaction. We speculate that the intramolecular interactions between the Src SH3 binding site and the FERM F1/F3 lobes may be an "autoinhibited" conformation that prevents binding of Src to FAK and therefore the full activation of FAK by phosphorylation of Y576, Y577, Y861, and Y925. Additionally, our structure implicates residues between the F1 and F3 lobes (I359, N339, M102, and H99) as potential sites of allosteric modulation as a means to relieve SH3 binding site-F1/F3 autoinhibitory interactions and allow Src binding.

\section{Conclusions}

In all, we present a higher resolution crystal structure of the FAK FERM domain that reveals novel information on the druggability of the Y397 FERM region and the intramolecular interactions of the Src SH3 binding site. These data may guide future drug discovery efforts in the development of FAK inhibitors that directly target the region proximal to key residue Y397. Also, this new crystal structure may serve to support future hypotheses investigating the structural regulation of FAK through modulation of the Src SH3 binding site.

\section{Methods}

Protein expression and purification

Avian FERM domain protein (residues 31-405) was expressed and purified similarly as previously reported [25]. The pET-chFERM construct was kindly provided by Dr. Michael J. Eck. In brief, BL21 (DE3) E. coli were transformed and subsequently cultured in LB media until an O.D. at $600 \mathrm{~nm}$ of 0.8 was reached. Protein expression was induced by addition of $0.2 \mathrm{mM}$ IPTG and grown in a shaking incubator for an additional $16 \mathrm{~h}$ at $30^{\circ} \mathrm{C}$. Cells were then pelleted and lysed with lysis buffer (20 mM Tris-Cl pH 8.0, $200 \mathrm{mM} \mathrm{NaCl}, 5 \mathrm{mM}$ BME, 10

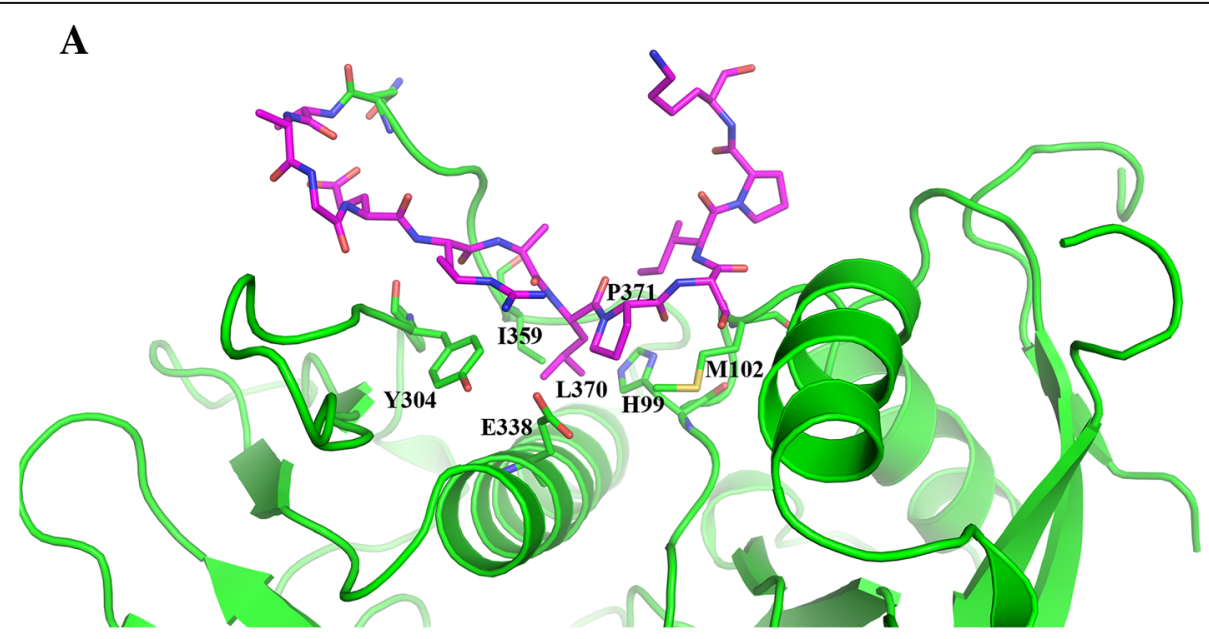

Fig. 4 Intramolecular Interactions of Src SH3 Binding Site. a Structural representation of the Src SH3 binding site (magenta) forming intramolecular interactions with a cleft in between the FERM F1 and F3 lobes (green). Note, key residues of contact are labeled 
mM imidazole, 1X Halt Protease Inhibitor Cocktail). Lysate was subjected to one freeze-thaw cycle followed by sonication for $2 \mathrm{~min}$ on ice. Supernatant was collected after centrifugation at 12,000 rpm for $15 \mathrm{~min}$ and incubated with $3 \mathrm{~mL} \mathrm{Ni-NTA}$ resin (Thermo) overnight. Resin was then washed with lysis buffer containing 25 $\mathrm{mM}$ imidazole and protein was eluted with lysis buffer containing $200 \mathrm{mM}$ imidazole.

The His-tag from the purified FERM-domain sample was removed by AcTEV-protease according to the manufacture protocol (Invitrogen). Briefly, 25 units of AcTEV-protease sample was enough to complete the cleavage of the His-tag from $1 \mathrm{mg}$ of the protein during overnight incubation at $40 \mathrm{C}$. The cleaved product was removed by filtration of the sample through Ni-NTA Superflow cartridge (5 ml, Qiagen) pre-equilibrated in $20 \mathrm{mM}$ Tris-HCL, pH 8.0, $100 \mathrm{mM} \mathrm{NaCl}, 1 \mathrm{mM}$ DTT and $20 \mathrm{mM}$ imidazole. The filtrated sample was loaded on a Q-Sepharose column (GE Healthcare) equilibrated in the same buffer except the imidazole and the protein was eluted using a linear gradient of $\mathrm{NaCl}$ from 0.1 to 1 $M$. The fractions of the His-off protein were pooled and applied on a Superdex 200 column (GE Healthcare) for further protein purification. The FERM-domain sample eluted from the gel filtration column was $95 \%$ pure as assessed by SDS-PAGE analysis and used for the crystallization experiments.

\section{Crystallization, data collection and structure determination}

Sitting drops in 96-round bottom well crystallization plates (Greiner Bio-One, GmbH, Germany) were set up using a Mosquito Robotic System (TTP LabTech, Hertfordshire, U.K.). Avian FAK FERM domain (residues 31405) protein sample at concentration of $10 \mathrm{mg} / \mathrm{ml}$ in 10 $\mathrm{mM}$ Tris- $\mathrm{HCl}$ buffer, $\mathrm{pH} 8.0,50 \mathrm{mM} \mathrm{NaCl}$ and $1 \mathrm{mM}$ DTT was used for the crystallization experiments.

Diffraction quality FERM protein crystals were obtained at $291 \mathrm{~K}$ from drops containing $0.5 \mu \mathrm{l}$ of the protein sample and $0.5 \mu \mathrm{l}$ of reservoir solution $(0.2 \mathrm{M} \mathrm{K} / \mathrm{Na}$ tartrate, 20\% PEG 3350). Diffraction data were collected at $100 \mathrm{~K}$ from a single, flash frozen, crystal [28], cryoprotected in $20 \%(\mathrm{v} / \mathrm{v})$ glycerol, on a Mar CCD-300 detector at LS-CAT (Advanced Photon Source, Argonne National Laboratory). Diffraction data were then indexed and processed with XDS software [29]. The crystal belonged to orthorhombic space group P212121 and contained two FERM molecules per asymmetric unit. The structure of FERM-domain was solved by molecular replacement using Phaser software [30] with the structure of avian FERM (residues 31-405) (PDB code 2AL6) as a search model. The final model of FERM-domain was progressively refined by performing several cycles of manual model building using COOT [31], subsequently followed by structure refinement with REFMAC [32]. Local NCS restraints were not used because of initial high resolution data. Crystallographic data and refinement statistics (including Ramachandran statistics) are shown in Table 1. Adequate side chain electron density was not found for residues E34, E93, E118, K141, K216, K218, K310, D311, R312, K313, E325, Q356, K364, E365, V391, S392, E393, and therefore they are not present in the final structure. Coordinates have been deposited in the Protein Data Bank with accession number 6CB0.

\section{In silico modeling}

The PyMOL molecular graphics program (Schrödinger, Inc.) was utilized for general structural representation of the FERM domain and publication-quality images were made using the ray-trace command. PyMOL was also used for sequence alignment and structural superposition using the align command. Multiple sequence alignment was performed using the Schrödinger Multiple Sequence Viewer application. For druggability prediction analysis of our crystal structure compared to previously published structures, we used the program SiteMap (Schrödinger, Inc.) as described [33]. SiteMap uses a grid-based searching algorithm, similar to the Goodford GRID algorithm [34]. We utilized the following settings: report up to 10 binding sites, a more restrictive definition of hydrophobicity, standard grid, and lower constraints to detect shallow binding sites. Identified binding sites with a calculated SiteScore $>0.8$ were classified as "druggable" sites having a high probability for sub-micromolar ligand binding.

\section{Abbreviations}

FAK: Focal Adhesion Kinase; FERM: Band 4.1, Ezrin, Radixin, Moesin; PDB: Protein Data Bank; SH3: Src Homology 3

\section{Acknowledgements}

This research used resources of the Advanced Photon Source, a U.S. Department of Energy (DOE) Office of Science User Facility operated for the DOE Office of Science by Argonne National Laboratory under Contract No. DE-AC02-06CH11357. Use of the LS-CAT Sector 21 was supported by the Michigan Economic Development Corporation and the Michigan Technology Tri-Corridor (Grant 085P1000817).

\section{Funding}

National Cancer Institute (grant No. R01 CA065910 to William Cance, Timothy Marlowe).

\section{Availability of data and materials}

The datasets generated during the current study are available in the Protein Data Bank (PDB) repository (PDB ID: 6CBO).

Authors' contributions

$T M, A D, S F$, and AR acquired and interpreted data; TM, MF, and WC supervised studies; TM and AD contributed to manuscript writing; all authors revised and approved the final manuscript.

Ethics approval and consent to participate Not applicable 


\section{Consent for publication}

Not applicable.

\section{Competing interests}

All authors have approved the manuscript and declare no conflict of interest.

\section{Publisher's Note}

Springer Nature remains neutral with regard to jurisdictional claims in published maps and institutional affiliations.

\section{Author details}

${ }^{1}$ Interdisciplinary Oncology, University of Arizona College of Medicine Phoenix, 475 N 5th Street, Phoenix, AZ 85004, USA. ²Pharmacology and Toxicology, University of Arizona College of Pharmacy, P.O. Box 210207, Tucson, AZ 85721, USA. ${ }^{3}$ Shamrock Structures, LLC, 1440 Davey Rd, Woodridge, IL 60517, USA. ${ }^{4}$ Structural Biology Center, Argonne Nationa Laboratory, 9700 South Cass Avenue, Argonne, IL 60439, USA. ${ }^{5}$ Neuro Oncology, Roswell Park Comprehensive Cancer Center, Elm \& Carlton Streets, Buffalo, NY 14263, USA. ${ }^{6}$ Cancer Center Division, University of Arizona Cancer Center, 625 N 6th Street, Phoenix, AZ 85004, USA.

Received: 20 March 2019 Accepted: 2 May 2019

Published online: 20 May 2019

\section{References}

1. Sulzmaier FJ, Jean C, Schlaepfer DD. FAK in cancer: mechanistic findings and clinical applications. Nat Rev Cancer. 2014;14(9):598-610.

2. Zhao J, Guan J-L. Signal transduction by focal adhesion kinase in cancer Cancer Metastasis Rev. 2009;28(1):35-49.

3. Weiner TM, Liu ET, Craven RJ, Cance WG. Expression of focal adhesion kinase gene and invasive cancer. Lancet. 1993;342(8878):1024-5.

4. Owens LV, Xu L, Craven RJ, Dent GA, Weiner TM, Kornberg L, Liu ET, Cance WG. Overexpression of the focal adhesion kinase (p125FAK) in invasive human tumors. Cancer Res. 1995:55(13):2752-5.

5. Golubovskaya VM, Kweh FA, Cance WG. Focal adhesion kinase and cancer. Histol Histopathol. 2009:24(4):503-10.

6. Schaller MD, Hildebrand JD, Shannon JD, Fox JW, Vines RR, Parsons JT. Autophosphorylation of the focal adhesion kinase, pp125FAK, directs SH2dependent binding of pp60src. Molecular \& Cellular Biology. 1994;14(3): 1680-8.

7. Marlowe TA, Lenzo FL, Figel SA, Grapes AT, Cance WG. Oncogenic receptor tyrosine kinases directly phosphorylate focal adhesion kinase (FAK) as a resistance mechanism to FAK-kinase inhibitors. Mol Cancer Ther. 2016: 15(12):3028-39.

8. Sieg DJ, Hauck CR, Ilic D, Klingbeil CK, Schaefer E, Damsky CH, Schlaepfer DD. FAK integrates growth-factor and integrin signals to promote cell migration. Nat Cell Biol. 2000;2(5):249-56.

9. Thomas JW, Ellis B, Boerner RJ, Knight WB, White GC 2nd, Schaller MD. SH2and $\mathrm{SH} 3$-mediated interactions between focal adhesion kinase and Src. J Biol Chem. 1998;273(1):577-83.

10. Hauck CR, Hunter T, Schlaepfer DD. The v-Src SH3 domain facilitates a cell adhesion-independent association with focal adhesion kinase. J Biol Chem. 2001;276(21):17653-62.

11. Westhoff MA, Serrels B, Fincham VJ, Frame MC, Carragher NO. SRC-mediated phosphorylation of focal adhesion kinase couples actin and adhesion dynamics to survival signaling. Mol Cell Biol. 2004;24(18):8113-33.

12. Frame MC, Patel $H$, Serrels $B$, Lietha D, Eck MJ. The FERM domain: organizing the structure and function of FAK. Nat Rev Mol Cell Biol. 2010; 11(11):802-14.

13. Cance WG, Kurenova E, Marlowe T, Golubovskaya V. Disrupting the scaffold to improve focal adhesion kinase-targeted cancer therapeutics. Sci Signal. 2013;6(268):pe10.

14. Golubovskaya VM, Finch R, Cance WG. Direct interaction of the N-terminal domain of focal adhesion kinase with the N-terminal transactivation domain of p53. J Biol Chem. 2005;280(26):25008-21.

15. Slack-Davis JK, Martin KH, Tilghman RW, Iwanicki M, Ung EJ, Autry C, Luzzio MJ, Cooper B, Kath JC, Roberts WG, et al. Cellular characterization of a novel focal adhesion kinase inhibitor. J Biol Chem. 2007;282(20):14845-52.

16. Roberts WG, Ung E, Whalen P, Cooper B, Hulford C, Autry C, Richter D, Emerson E, Lin J, Kath J, et al. Antitumor activity and pharmacology of a selective focal adhesion kinase inhibitor, PF-562,271. Cancer Res. 2008;68(6): 1935-44.

17. Tanjoni I, Walsh C, Uryu S, Tomar A, Nam JO, Mielgo A, Lim ST, Liang C, Koenig M, Sun C, et al. PND-1186 FAK inhibitor selectively promotes tumor cell apoptosis in three-dimensional environments. Cancer Biol Ther. 2010; 9(10):764-77.

18. Shi Q, Hjelmeland AB, Keir ST, Song L, Wickman S, Jackson D, Ohmori O, Bigner DD, Friedman HS, Rich JN. A novel low-molecular weight inhibitor of focal adhesion kinase, TAE226, inhibits glioma growth. Mol Carcinog. 2007; 46(6):488-96.

19. Infante JR, Camidge DR, Mileshkin LR, Chen EX, Hicks RJ, Rischin D, Fingert H, Pierce KJ, Xu H, Roberts WG, et al. Safety, pharmacokinetic, and pharmacodynamic phase I dose-escalation trial of PF-00562271, an inhibitor of focal adhesion kinase, in advanced solid tumors. J Clin Oncol. 2012; 30(13):1527-33.

20. Verastem. Placebo Controlled Study of VS-6063 in Subjects With Malignant Pleural Mesothelioma (COMMAND) [https:/clinicaltrials.gov/ct2/show/study/ NCT01870609] Accessed 24 Apr 2016

21. Golubovskaya VM, Nyberg C, Zheng M, Kweh F, Magis A, Ostrov D, Cance WG. A small molecule inhibitor, 1,2,4,5-benzenetetraamine tetrahydrochloride, targeting the $\mathrm{Y} 397$ site of focal adhesion kinase decreases tumor growth. J Med Chem. 2008:51:7405-16.

22. Akl MR, Foudah Al, Ebrahim HY, Meyer SA, El Sayed KA. The marine-derived sipholenol A-4-O-3',4'-dichlorobenzoate inhibits breast cancer growth and motility in vitro and in vivo through the suppression of Brk and FAK signaling. Mar Drugs. 2014;12(4):2282-304.

23. Thiyagarajan V, Lin SH, Chia YC, Weng CF. A novel inhibitor, 16-hydroxycleroda-3,13-dien-16,15-olide, blocks the autophosphorylation site of focal adhesion kinase (Y397) by molecular docking. Biochim Biophys Acta. 2013; 1830(8):4091-101.

24. Golubovskaya VM, Figel S, Ho BT, Johnson CP, Yemma M, Huang G, Zheng M, Nyberg C, Magis A, Ostrov DA, et al. A small molecule focal adhesion kinase (FAK) inhibitor, targeting Y397 site: 1-(2-hydroxyethyl)-3, 5, 7-triaza-1azoniatricyclo [3.3.1.1(3,7)]decane; bromide effectively inhibits FAK autophosphorylation activity and decreases cancer cell viability, clonogenicity and tumor growth in vivo. Carcinogenesis. 2012;33(5):1004-13.

25. Ceccarelli DF, Song HK, Poy F, Schaller MD, Eck MJ. Crystal structure of the FERM domain of focal adhesion kinase. J Biol Chem. 2006;281(1):252-9.

26. Brami-Cherrier K, Gervasi N, Arsenieva D, Walkiewicz K, Boutterin MC, Ortega A, Leonard PG, Seantier B, Gasmi L, Bouceba T, et al. FAK dimerization controls its kinase-dependent functions at focal adhesions. EMBO J. 2014;33(4):356-70.

27. Brunger AT. Free $R$ value: a novel statistical quantity for assessing the accuracy of crystal structures. Nature. 1992;355(6359):472-5.

28. Teng T-Y. Mounting of crystals for macromolecular crystallography in a freestanding thin film. J Appl Crystallogr. 1990;23:387-91.

29. Kabsch W. Xds. Acta Crystallogr D Biol Crystallogr. 2010;66(Pt 2):125-32.

30. McCoy AJ, Grosse-Kunstleve RW, Adams PD, Winn MD, Storoni LC, Read RJ. Phaser crystallographic software. J Appl Crystallogr. 2007;40:658-74.

31. Emsley P, Lohkamp B, Scott WG, Cowtan K. Features and development of Coot. Acta Crystallogr D Biol Crystallogr. 2010;66(Pt 4):486-501.

32. Murshudov GN, Vagin AA, Dodson EJ. Refinement of macromolecular structures by the maximum-likelihood method. Acta Crystallogr D Biol Crystallogr. 1997;53(Pt 3:240-55.

33. Halgren TA. Identifying and characterizing binding sites and assessing druggability. J Chem Inf Model. 2009;49(2):377-89.

34. Goodford PJ. A computational procedure for determining energetically favorable binding sites on biologically important macromolecules. J Med Chem. 1985;28(7):849-57.

Ready to submit your research? Choose BMC and benefit from:

- fast, convenient online submission

- thorough peer review by experienced researchers in your field

- rapid publication on acceptance

- support for research data, including large and complex data types

- gold Open Access which fosters wider collaboration and increased citations

- maximum visibility for your research: over $100 \mathrm{M}$ website views per year

At $\mathrm{BMC}$, research is always in progress.

Learn more biomedcentral.com/submission 